\title{
Time-resolved transcriptional profiling of Trichinella-infected murine myocytes helps to elucidate host-pathogen interactions in the muscle stage
}

Xiaoxiang $\mathrm{Hu}^{1 \dagger}$, Xiaolei $\mathrm{Liu}^{1 \dagger}$, Chen $\mathrm{Li}^{1 \dagger}$, Yulu Zhang ${ }^{1}$, Chengyao $\mathrm{Li}^{1}$, Yanfeng $\mathrm{Li}^{1}$, Yingxi Chen ${ }^{1}$, Heng Guo ${ }^{2}$, Xue Bai ${ }^{{ }^{*}}$ and Mingyuan Liu ${ }^{1,3^{*}}$

\begin{abstract}
Background: Parasites of the genus Trichinella are the pathogenic agents of trichinellosis, which is a widespread and severe foodborne parasitic disease. Trichinella spiralis resides primarily in mammalian skeletal muscle cells. After invading the cells of the host organism, T. spiralis must elude or invalidate the host's innate and adaptive immune responses to survive. It is necessary to characterize the pathogenesis of trichinellosis to help to prevent the occurrence and further progression of this disease. The aims of this study were to elucidate the mechanisms of nurse cell formation, pathogenesis and immune evasion of T. spiralis, to provide valuable information for further research investigating the basic cell biology of Trichinella-infected muscle cells and the interaction between T. spiralis and its host.
\end{abstract}

Methods: We performed transcriptome profiling by RNA sequencing to identify global changes at 1, 3, 7, 10 and 15 days post-infection (dpi) in gene expression in the diaphragm after the parasite entered and persisted within the murine myocytes; the mice were infected by intravenous injection of newborn larvae. Gene expression analysis was based on the alignment results. Differentially expressed genes (DEGs) were identified based on their expression levels in various samples, and functional annotation and enrichment analysis were performed.

Results: The most extensive and dynamic gene expression responses in host diaphragms were observed during early infection (1 dpi). The number of DEGs and genes annotated in the Kyoto Encyclopedia of Genes and Genomes and Gene Ontology databases decreased significantly in the infected mice compared to the uninfected mice at 3 and $7 \mathrm{dpi}$, suddenly increased sharply at $10 \mathrm{dpi}$, and then decreased to a lower level at $15 \mathrm{dpi}$, similar to that observed at 3 and $7 \mathrm{dpi}$. The massive initial reaction of the murine muscle cells to Trichinella infection steadied in the later stages of infection, with little additional changes detected for the remaining duration of the studied process. Although there were hundreds of DEGs at each time point, only 11 genes were consistently up- or downregulated at all 5 time points. Conclusions: The gene expression patterns identified in this study can be employed to characterize the coordinated response of T. spiralis-infected myocytes in a time-resolved manner. This comprehensive dataset presents a distinct and sensitive picture of the interaction between host and parasite during intracellular infection, which can help to

\footnotetext{
*Correspondence: namiya23@163.com; liumy36@163.com

${ }^{\dagger}$ Xiaoxiang Hu, Xiaolei Liu and Chen Li contributed equally to this work

${ }^{1}$ Key Laboratory of Zoonosis Research, Ministry of Education,

Institute of Zoonosis, College of Veterinary Medicine, Jilin University,

Changchun 130062, China

Full list of author information is available at the end of the article
}

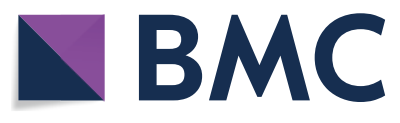

(c) The Author(s) 2021. This article is licensed under a Creative Commons Attribution 4.0 International License, which permits use, sharing, adaptation, distribution and reproduction in any medium or format, as long as you give appropriate credit to the original author(s) and the source, provide a link to the Creative Commons licence, and indicate if changes were made. The images or other third party material in this article are included in the article's Creative Commons licence, unless indicated otherwise in a credit line to the material. If material is not included in the article's Creative Commons licence and your intended use is not permitted by statutory regulation or exceeds the permitted use, you will need to obtain permission directly from the copyright holder. To view a copy of this licence, visit http://creativeco mmons.org/licenses/by/4.0/. The Creative Commons Public Domain Dedication waiver (http://creativecommons.org/publicdomain/ zero/1.0/) applies to the data made available in this article, unless otherwise stated in a credit line to the data. 
elucidate how pathogens evade host defenses and coordinate the biological functions of host cells to survive in the mammalian environment.

Keywords: Trichinella spiralis, Transcriptome, RNA sequencing, Host-pathogen interplay

\section{Background}

Trichinellosis is a widespread and severe foodborne parasitic disease caused by the consumption of raw or undercooked pork or its products which contain infective larvae of Trichinella nematodes [1]. Trichinella spiralis, an intracellular parasitic nematode, is capable of infecting more than 150 species of animals, including humans [2]. The life cycle of T. spiralis comprises three stages: newborn larvae (NBL), muscle larvae (ML) and adult worms [3]. After infection, the infective ML molt into adults, mate and reproduce. Next, the cyetic females release NBL, which migrate and invade skeletal myocytes. NBL grow rapidly, cause extensive damage to host myocytes and transform them into a new form, known as nurse cells [4]. Infection with $T$. spiralis can trigger a series of dynamic reactions in the host, resulting in changes in gene expression patterns in muscle, which enable T. spiralis to successfully colonize the body. It has been reported that some of the events that occur during nurse cell formation happen in parallel with those occurring during myocyte regeneration/repair [5]. The process of nurse cell formation is accompanied by many biological events in the myocyte, including the activation and proliferation of satellite cells, muscle damage and regeneration, redifferentiation and transformation of the infected muscle cells, and apoptosis leading to changes in the cytoplasm (replacement of basophilic cytoplasm, which originates from the damaged muscle cell, by eosinophilic cytoplasm, which originates from proliferative satellite cells). Infection by $T$. spiralis causes nuclear hypertrophy; these nuclei have been shown to have a mean DNA content of $4 \mathrm{~N}$, which implies long-term arrest of the cycle of the infected cell at G2/M [6]. In addition, the synthesis of type I, IV, and VI collagen, which are crucial components of the nurse cell collagen capsule, significantly increase during T. spiralis infection [7].

To search for the candidate genes responsible for the formation of nurse cells, a cDNA microarray analysis was performed on muscle tissues sampled before and after Trichinella infection, to show the kinetics of the genes mobilized in response to Trichinella infection and provide clues regarding candidate genes in muscle conversion [8]. More recently, researchers analyzed the changes in primary myoblasts induced by ML excretory-secretory products. Microarray analyses disclosed expression variations in muscle cell differentiation, proliferation, cytoskeleton organization, cell motion, transcription, cell cycle, apoptosis and signaling pathways, such as the mitogen-activated protein kinase, Janus kinase/signal transducers and activators of transcription, Wingless/int1 and phosphatidylinositol 3 kinase/protein kinase B pathways, thereby providing novel information regarding how $T$. spiralis can modify the standard course of skeletal muscle renovation after NBL aggression to achieve nurse cell formation [9].

To analyze the interaction between T. spiralis and host myocytes in greater depth at different time points during T. spiralis development after invasion, we conducted a comprehensive and in-depth host transcriptome study after transcriptome sequencing of $T$. spiralis by using advanced RNA sequencing (RNA-seq) technology. Unlike the genome, which has a static state and exhibits relative stability, the transcriptome can change with the development of the species itself or with changes in external environmental conditions. The gene expression of the host may vary considerably in different developmental stages or environments. Therefore, the transcriptome has strong temporal and spatial characteristics. Gene transcription levels change as a result of a dynamic relationship between the host genome and external physical characteristics, and can reflect a given developmental or physiological stage. Hence we can obtain a stronger understanding of the characteristics and regulatory mechanisms of a parasite by characterizing the changes in the transcriptome of its host. For example, mouse genome-wide transcriptional responses to infection with two strains of Plasmodium yoelii were investigated and deviations in innate response approaches matching strain-specific disease phenotypes were elucidated, demonstrating that a robust type I interferon response contributed to a decrease in parasitemia in N67-infected mice [10]. An increasing number of studies have indicated the value of studying host transcriptome changes to elucidate host-parasite interactions.

A global and kinetic analysis of gene expression profiles of $T$. spiralis-infected muscle with RNA-seq were performed in this study. The time points $(1,3,7,10$ and 15 days post-intravenous injection of NBL, which are equivalent to about $7,10,13,16$ and 21 days post-oral infection) of observation correspond to the duration of nurse cell formation. This study attempted to elucidate the mechanisms of nurse cell formation, pathogenesis and immune evasion, to provide valuable information for further research investigating the basic cell biology 
of Trichinella-infected muscle cells and the interaction between T. spiralis and its host. Analyzing differentially expressed genes (DEGs) and pathways may help to establish a foundation for determining the invasion and parasitic mechanisms employed by $T$. spiralis, and may have significance for pathological and immunological research on T. spiralis and the screening of therapeutic drugs for trichinellosis.

\section{Methods}

\section{Mice and $T$. spiralis infection}

$\mathrm{BALB} / \mathrm{c}$ mice (6-8 weeks old) were obtained from the Experimental Animal Center of the College of Basic Medical Sciences, Jilin University (Changchun, China). To obtain more significant transcriptome data, synchronized $T$. spiralis infections were initiated with NBL produced by adult female parasites in vitro, as described previously [11]. Mice injected with approximately 40,000 NBL each via tail vein were sacrificed at $1,3,7,10$ and 15 dpi. Uninfected mice ( $0 \mathrm{dpi})$ and NBL that were not injected into mice were employed as controls. There were three biological replicates for each group at every time point.

\section{RNA extraction and qualification}

Total RNA was extracted from mice diaphragms or NBL using RNAiso Plus (TaKaRa) according to the manufacturer's recommendations. RNA concentration and purity were determined by NanoDrop 2000 (Thermo Fisher Scientific, Wilmington, DE). RNA integrity was evaluated using the RNA Nano 6000 Assay Kit of the Agilent Bioanalyzer 2100 system (Agilent Technologies, CA, USA).

\section{Library preparation for transcriptome sequencing}

A total of $1 \mu \mathrm{g}$ RNA per sample was used as input material for the RNA sample preparation. Sequencing libraries were generated using the NEBNext Ultra RNA Library Prep Kit for Illumina (NEB, USA) following the manufacturer's instructions, and index codes were added to attribute sequences to each sample. Briefly, mRNA was purified from total RNA using poly-T oligoattached magnetic beads. Fragmentation was conducted using divalent cations under elevated temperature in NEBNext First Strand Synthesis Reaction Buffer (5X). First-strand cDNA was synthesized using random hexamer primers and M-MuLV reverse transcriptase. Second-strand cDNA synthesis was subsequently performed employing DNA polymerase I and RNase $H$. The remaining overhangs were converted into blunt ends via exonuclease/polymerase activities. After adenylation of the $3^{\prime}$ ends of DNA fragments, NEBNext adaptors with hairpin loop structures were ligated for hybridization preparation. To select cDNA fragments preferentially measuring $240 \mathrm{bp}$ in length, the library fragments were purified with the AMPure XP system (Beckman Coulter, Beverly, MA). Then, $3 \mu$ l USER Enzyme (NEB, USA) was utilized with size-selected, adaptor-ligated cDNA at $37{ }^{\circ} \mathrm{C}$ for $15 \mathrm{~min}$ followed by $5 \mathrm{~min}$ at $95{ }^{\circ} \mathrm{C}$ before polymerase chain reaction (PCR) analysis. Then, PCR was performed with Phusion High-Fidelity DNA polymerase, universal PCR primers and Index (X) primer. Finally, PCR products were purified (AMPure XP system) and library quality was assessed using the Agilent Bioanalyzer 2100 system.

\section{Clustering and sequencing}

The clustering of the index-coded samples was performed on a cBot Cluster Generation System using TruSeq PE Cluster Kit v4-cBot-HS (Illumina) according to the manufacturer's instructions. After cluster generation, the library preparations were sequenced on an Illumina platform and paired-end reads were generated.

\section{Quality control}

Raw data (raw reads) in fastq format were first processed through in-house Perl scripts. In this step, clean data (clean reads) were obtained by removing low-quality reads and reads containing the adaptor, poly- $\mathrm{N}$, from raw data. In the meantime, the Q20, Q30, GC content and sequence duplication level of the clean data were calculated. All downstream analyses were based on clean, high-quality data.

\section{Comparative analysis}

Clean reads were subsequently mapped to the reference genome sequence (GRCm38_release95). Only reads with a perfect match or one mismatch were further analyzed and annotated based on the reference genome. Hisat2 tools software was used for mapping with the mouse reference genome.

\section{Gene functional annotation}

Gene function was annotated based on the following databases: National Center for Biotechnology Information [non-redundant protein sequences $(\mathrm{Nr})$; non-redundant nucleotide sequences (Nt)]; Pfam (protein families); Eukaryotic Orthologous Groups/Clusters of Orthologous Groups (KOG/COG); Swiss-Prot (a manually annotated and reviewed protein sequence database); Kyoto Encyclopedia of Genes and Genomes (KEGG) ortholog database (KO); Gene Ontology (GO).

\section{Quantification of gene expression levels}

Fragments per kilobase of transcript per million fragments mapped reads (FPKM) were used to quantify the gene expression levels. The formula is as follows: 


$$
\text { FPKM }=\frac{\text { cDNA Fragments }}{\text { Mapped Fragments (Millions) } * \text { Transcript Length }(\mathrm{kb})}
$$

\section{Differential expression analysis}

Differential expression analysis of the two groups was performed employing edgeR. Statistical routines for determining differential expression in digital gene expression data were provided by edgeR using a model based on the negative binomial distribution. The resulting $p$-values were adjusted by using Benjamini and Hochberg's approach for controlling the false discovery rate. Genes with adjusted $p$-values $<0.01$, as determined by edgeR, were considered to be differentially expressed.

\section{KEGG pathway enrichment analysis}

KEGG is a database that can be used to understand the high-level functions and utilities of biological systems, such as cells, organisms and ecosystems, from molecular-level information, especially large-scale molecular datasets generated by genome sequencing and other high-throughput experimental technologies (http://www.genome.jp/kegg/). We tested the statistical enrichment of DEGs in KEGG pathways employing KEGG Orthology Based Annotation System software.

\section{GO enrichment analysis}

GO enrichment analysis of the DEGs was performed by the GOseq R package-based Wallenius' noncentral hypergeometric distribution, which can adjust for gene length bias in DEGs.

\section{Verification of specific transcripts by quantitative real-time PCR}

To verify the expression levels of mRNA, reverse transcription was performed using the QuantiNova Reverse Transcription Kit (QIAGEN, Germany) according to the supplier's recommendations. The resulting cDNA was analyzed by quantitative real-time PCR using an Applied Biosystems StepOnePlus Real-Time PCR System (Thermo Fisher Scientific, USA). The primer sequences utilized in this study are listed in Table 1. Each sample was assayed in triplicate wells on the same plate for all genes. Each reaction contained FastStart Essential DNA Green Master (Roche, Germany), forward primer $(0.2 \mu \mathrm{M})$, reverse primer $(0.2 \mu \mathrm{M})$, and cDNA template to a final volume of $20 \mu \mathrm{l}$ in nucleasefree water. Amplification of all genes was performed under the following conditions: a holding time of $95^{\circ} \mathrm{C}$ for $10 \mathrm{~min}$ followed by 40 cycles of denaturation at $95{ }^{\circ} \mathrm{C}$ for $15 \mathrm{~s}$ and primer annealing at $59{ }^{\circ} \mathrm{C}$ for $30 \mathrm{~s}$.
Table 1 Primer sequences used for the quantitative real-time polymerase chain reaction

\begin{tabular}{ll}
\hline Gene & Primer sequence (5' $\rightarrow$ 3') \\
\hline GAPDH & Forward 5'-TACCCCCAATGTGTCCGTC-3' \\
Ciart & Reverse 5'-AAGAGTGGGAGTTGCTGTTGAAG-3' \\
& Forward 5'-GTTGCATCCTATGTCCGCCTGTC-3' \\
Per2 & Reverse 5'-TGGCTAGTCATCTGTGGCTCTGG-3' \\
& Forward 5'-GAAACTGAAGTCAAAACGCGTC-3' \\
Serpine1 & Reverse 5'-GTCCCTGGTGTGGATACTATTC-3' \\
Gpnmb & Forward 5'-ATCTTGGATGCTGAACTCATCA-3' \\
Ankrd1 & Reverse 5'-GAGAGAACTTAGGCAGGATGAG-3' \\
& Forward 5'-GATCTCTATCCCTGGCAAAGAC-3' \\
Cilp2 & Reverse 5'-CAGTTTCCTATTGGCTTGTACG-3' \\
& Forward 5'-GCTGTGAGGCTGAACCGCTATAAG-3' \\
Tnmd & Reverse 5'-CCAGCACAGTTCTTGACCTTGAGG-3' \\
& Forward 5'-GACAAGTACGAGTACGACGTG-3 \\
Col2a1 & Reverse 5'-TTGTGTGAACGCACCATGTA-3' \\
& Forward 5'-GAACAGTCAGTGATTTGGTTC-3' \\
Wif1 & Reverse 5'-GGTCACATTATCGCAAATCTCC-3' \\
& Forward 5'-TACTGGAGTGACTGGTCCTAAG-3' \\
& Reverse 5'-AACACCTTTGGGACCATCTTTT-3' \\
& Forward 5'-GAAATGGAGGCTTTTGTAACGA-3' \\
& Reverse 5'-GCAAATACATTTTCCCGGTAA-3' \\
\hline
\end{tabular}

GADPH Glyceraldehyde-3-phosphate dehydrogenase; Ciart circadian-associated repressor of transcription; Per2 period circadian clock 2; Serpine1 serine (or cysteine) peptidase inhibitor, clade E, member 1; Gpnmb glycoprotein (transmembrane) nmb; Ankrd1 ankyrin repeat domain 1 (cardiac muscle); Cilp2 cartilage intermediate layer protein 2; Tnmd tenomodulin; Col2a1 collagen, type II, alpha 1; Wif1 Wnt inhibitory factor 1

mRNA fold expression values were analyzed using the relative threshold cycle $(\mathrm{Ct})\left(2^{-\Delta \Delta \mathrm{Ct}}\right)$ method $[12,13]$.

\section{Statistical analysis}

Statistical analysis was performed using GraphPad Prism 8 software for Windows. Comparisons among the treatment and control groups were analyzed using one-way ANOVA. Data are expressed as the mean \pm SD of three replicate groups.

\section{Results}

Infection dynamics and global transcription patterns

Transcriptome profiling using RNA-seq was employed to identify global variations in gene expression over the course of infection of murine diaphragms with $T$. spiralis. Over 1.5 billion sequence reads were assembled across three independent experiments (biological 
Table 2 Sequencing data statistics

\begin{tabular}{|c|c|c|c|c|}
\hline Samples & Clean reads $^{\mathrm{a}}$ & Clean bases $^{b}$ & GC content ${ }^{\mathrm{C}}(\%)$ & $\% \geq{\mathrm{Q} 30^{\mathrm{d}}}^{\mathrm{d}}$ \\
\hline$d-0-1$ & $29,306,627$ & $8,723,905,770$ & 49.41 & 93.33 \\
\hline$d-0-2$ & $29,701,695$ & $8,856,713,318$ & 49.12 & 93.23 \\
\hline$d-0-3$ & $24,573,915$ & $7,325,026,546$ & 49.13 & 93.51 \\
\hline$d-1-1$ & $42,774,938$ & $12,743,577,440$ & 49.50 & 95.06 \\
\hline$d-1-2$ & $47,356,896$ & $14,119,786,038$ & 49.09 & 94.64 \\
\hline$d-1-3$ & $47,632,923$ & $14,229,261,830$ & 49.12 & 94.83 \\
\hline$d-3-1$ & $41,692,065$ & $12,459,755,692$ & 49.22 & 94.96 \\
\hline$d-3-2$ & $44,962,040$ & $13,435,712,256$ & 49.19 & 95.19 \\
\hline$d-3-3$ & $41,808,804$ & $12,496,583,212$ & 49.03 & 95.04 \\
\hline$d-7-1$ & $52,403,887$ & $15,659,838,448$ & 48.70 & 94.91 \\
\hline$d-7-2$ & $44,892,431$ & $13,419,760,674$ & 49.43 & 94.82 \\
\hline$d-7-3$ & $43,566,080$ & $13,011,606,674$ & 48.79 & 94.77 \\
\hline$d-10-1$ & $46,072,970$ & $13,783,739,164$ & 49.41 & 94.85 \\
\hline$d-10-2$ & $53,341,148$ & $15,929,948,426$ & 49.46 & 94.83 \\
\hline$d-10-3$ & $50,069,261$ & $14,950,813,380$ & 49.36 & 94.67 \\
\hline$d-15-1$ & $47,120,191$ & $14,081,050,788$ & 49.71 & 94.60 \\
\hline$d-15-2$ & $41,755,099$ & $12,464,431,734$ & 48.94 & 94.73 \\
\hline$d-15-3$ & $42,645,130$ & $12,738,561,562$ & 49.23 & 94.92 \\
\hline
\end{tabular}

${ }^{a}$ Paired-end reads of clean data

b Total number of bases of clean data

' $\mathrm{GC}$ content percentage of clean data, i.e. percentage of clean data for bases G and C

${ }^{d}$ Percentage of bases with a Q-score of $\geq 30$

replicates) and labeled batches 1-3. We obtained $230.43 \mathrm{~Gb}$ of clean data after mRNA sequencing for 18 samples, with at least $7.33 \mathrm{~Gb}$ clean data for each sample, and a quality score of $93.23 \%$, i.e. percentage of bases with a $Q$ score of $\geq 30$, where a score of 30 represents an error rate of 1 in 1000 (see Table 2). Next, the clean data were mapped to the reference genome, with the mapping ratio varying from $95.02 \%$ to $97.27 \%$ (see Table 3).

Seeing that there is tiny sequence preservation between mouse and T. spiralis and a notably low percentage of T. spiralis mapped reads in the clean reads (the highest is merely $1.25 \%$; unpublished data), we were capable of explicitly mapping reads from mouse RNAs from the compound samples to its genome. For each time point, the percentage of reads mapping to the host changed only slightly over the course of the infection. Although there was no significant difference, there was a slight upward and then downward trend in the percentage of murine mapped reads as the infection progressed (Fig. 1). This tiny nonuniformity was observed for the percentage of reads mapping to mouse in all samples, which may help to characterize the variable transcriptional activity of the host. A possible explanation for this phenomenon is that mouse diaphragm cells were more transcriptionally active when first stimulated by the parasite and became less transcriptionally active as the infection progressed.

\section{Statistical assessment of biological replicates}

Pearson's correlation coefficient $\left(R^{2}\right)$ is a commonly used evaluation index of correlation between biological replicates [14]. The closer the $R^{2}$ is to 1 , the more similar the two replicates. We employed a correlation heatmap to visualize the relationship between the experimental datasets (Fig. 2), and found that $R^{2}$ between most biological replications was approximately 0.9. This analysis demonstrated that there was a high level of repeatability between the biological replicates exposed to the same experimental conditions (dpi).

\section{Identification of DEGs in diaphragms}

While the heatmap analyses gave an advanced summary of the behavior of the transcriptomes of the murine diaphragms during infection, further investigation was required to assess alterations in the expression levels of individual genes. A differential expression analysis was performed to closely examine the response of the murine diaphragms to $T$. spiralis infection at $0,1,3,7,10$ and 15 dpi. Pairwise comparison within and across individual time points were carried out to compare infected with uninfected diaphragms (Figs. 3, 4).

The most extensive response to infection was acquired at $1 \mathrm{dpi}$, with 1013 DEGs between the uninfected and infected diaphragms. There was a lesser response at later time points, as reflected by fewer DEGs: 193, 82, 504 and 97 genes at 3, 7, 10 and $15 \mathrm{dpi}$, respectively. The analysis of up- and downregulated genes for each time point comparison is depicted in Fig. 4 (top panel). Complete DEG lists for each time point are provided in Additional file 1: Table S1. Other than a slight upward and then downward trend in the percentage of mapped reads as the infection progressed as previously mentioned, the number of DEGs in the infected compared to the uninfected diaphragms decreased significantly at 3 and $7 \mathrm{dpi}$, suddenly increased sharply at $10 \mathrm{dpi}$, then dropped to a lower level at $15 \mathrm{dpi}$, which was similar to that observed at 3 and 7 dpi.

An immediate comparison using the superposition of DEGs at each time point demonstrated that the diaphragm response to infection at $1 \mathrm{dpi}$ was notably different to that at the subsequent time points (Fig. 3). Of the 1013 DEGs at $1 \mathrm{dpi}, 77.8 \%$ (788 genes) were unique. In contrast, $31.1 \%$ of genes at $3 \mathrm{dpi}(60), 19.5 \%$ of genes at $7 \mathrm{dpi}$ [16], 58.9\% of genes at $10 \mathrm{dpi}$ (297) and $28.9 \%$ of genes at $15 \mathrm{dpi}$ [28] were differentially expressed only at that specific time point. Higher percentages of unique genes were still observed at 1 and $10 \mathrm{dpi}$. Indeed, only 11 
Table 3 Alignment results of each sample

\begin{tabular}{|c|c|c|c|c|c|c|}
\hline Samples & Total reads ${ }^{\mathrm{a}}$ & Mapped reads ${ }^{b}$ & Uniq. mapped reads ${ }^{c}$ & Multiple map. reads ${ }^{d}$ & Reads map to' $+^{\prime e}$ & Reads map to'- $-^{f}$ \\
\hline$d-0-1$ & $58,613,254$ & $56,475,681$ (96.35\%) & $50,859,528(86.77 \%)$ & $5,616,153(9.58 \%)$ & $25,981,307$ (44.33\%) & $26,076,507$ (44.49\%) \\
\hline$d-0-2$ & $59,403,390$ & $57,299,237(96.46 \%)$ & $51,049,269(85.94 \%)$ & $6,249,968$ (10.52\%) & $26,071,598$ (43.89\%) & $26,139,723(44.00 \%)$ \\
\hline$d-0-3$ & $49,147,830$ & $47,414,092(96.47 \%)$ & $42,500,765(86.48 \%)$ & $4,913,327(10.00 \%)$ & $21,732,380(44.22 \%)$ & $21,786,530(44.33 \%)$ \\
\hline$d-1-1$ & $85,549,876$ & $82,325,825$ (96.23\%) & $74,360,476$ (86.92\%) & $7,965,349(9.31 \%)$ & $38,336,236$ (44.81\%) & $38,490,739$ (44.99\%) \\
\hline$d-1-2$ & $94,713,792$ & $91,639,498$ (96.75\%) & $81,994,693$ (86.57\%) & $9,644,805$ (10.18\%) & $42,254,746$ (44.61\%) & $42,390,965$ (44.76\%) \\
\hline$d-1-3$ & $95,265,846$ & $92,379,516(96.97 \%)$ & $81,663,964$ (85.72\%) & $10,715,552(11.25 \%)$ & $42,047,639(44.14 \%)$ & $42,154,318(44.25 \%)$ \\
\hline$d-3-1$ & $83,384,130$ & $80,785,406$ (96.88\%) & $71,651,204(85.93 \%)$ & $9,134,202(10.95 \%)$ & $36,970,139(44.34 \%)$ & $37,058,529(44.44 \%)$ \\
\hline$d-3-2$ & $89,924,080$ & $87,153,989(96.92 \%)$ & $77,696,003(86.40 \%)$ & $9,457,986$ (10.52\%) & $39,952,614(44.43 \%)$ & $40,064,092(44.55 \%)$ \\
\hline$d-3-3$ & $83,617,608$ & $81,334,162(97.27 \%)$ & $71,806,277$ (85.87\%) & 9,527,885 (11.39\%) & $36,949,431$ (44.19\%) & $37,014,840(44.27 \%)$ \\
\hline$d-7-1$ & $104,807,774$ & $101,706,283(97.04 \%)$ & $89,439,347$ (85.34\%) & $12,266,936(11.70 \%)$ & $46,119,278(44.00 \%)$ & $46,223,712(44.10 \%)$ \\
\hline$d-7-2$ & $89,784,862$ & $86,469,953$ (96.31\%) & $78,051,494$ (86.93\%) & $8,418,459(9.38 \%)$ & $40,087,913(44.65 \%)$ & $40,143,735(44.71 \%)$ \\
\hline$d-7-3$ & $87,132,160$ & $84,256,540$ (96.70\%) & $74,469,393$ (85.47\%) & $9,787,147$ (11.23\%) & $38,286,864$ (43.94\%) & $38,382,981$ (44.05\%) \\
\hline$d-10-1$ & $92,145,940$ & $89,221,639(96.83 \%)$ & $81,900,144(88.88 \%)$ & $7,321,495$ (7.95\%) & $42,120,068(45.71 \%)$ & $42,160,622(45.75 \%)$ \\
\hline$d-10-2$ & $106,682,296$ & $102,682,416(96.25 \%)$ & $92,480,400(86.69 \%)$ & $10,202,016(9.56 \%)$ & $47,660,016(44.67 \%)$ & $47,779,175$ (44.79\%) \\
\hline$d-10-3$ & $100,138,522$ & $96,653,596(96.52 \%)$ & $87,175,782(87.06 \%)$ & $9,477,814(9.46 \%)$ & $44,725,983(44.66 \%)$ & $44,860,821(44.80 \%)$ \\
\hline$d-15-1$ & $94,240,382$ & $90,680,881$ (96.22\%) & $80,900,655$ (85.84\%) & $9,780,226(10.38 \%)$ & $41,755,839(44.31 \%)$ & $41,819,200(44.38 \%)$ \\
\hline$d-15-2$ & $83,510,198$ & $79,354,215$ (95.02\%) & $70,468,299(84.38 \%)$ & $8,885,916(10.64 \%)$ & $36,144,364(43.28 \%)$ & $36,222,735(43.38 \%)$ \\
\hline$d-15-3$ & $85,290,260$ & $81,572,791$ (95.64\%) & $73,292,073$ (85.93\%) & $8,280,718(9.71 \%)$ & $37,623,305(44.11 \%)$ & $37,700,361$ (44.20\%) \\
\hline
\end{tabular}

a Number of reads of clean data, not paired-end reads

b Number of reads mapped to the reference genome and the percentage in clean reads

c Number of reads mapped uniquely (Uniq.) to the reference genome and the percentage in clean reads

${ }^{d}$ Number of reads multiply mapped (map.) to the reference genome and the percentage in clean reads

e Number of reads mapped to the sense chain and the percentage in clean reads

f Number of reads mapped to the anti-sense chain and the percentage in clean reads

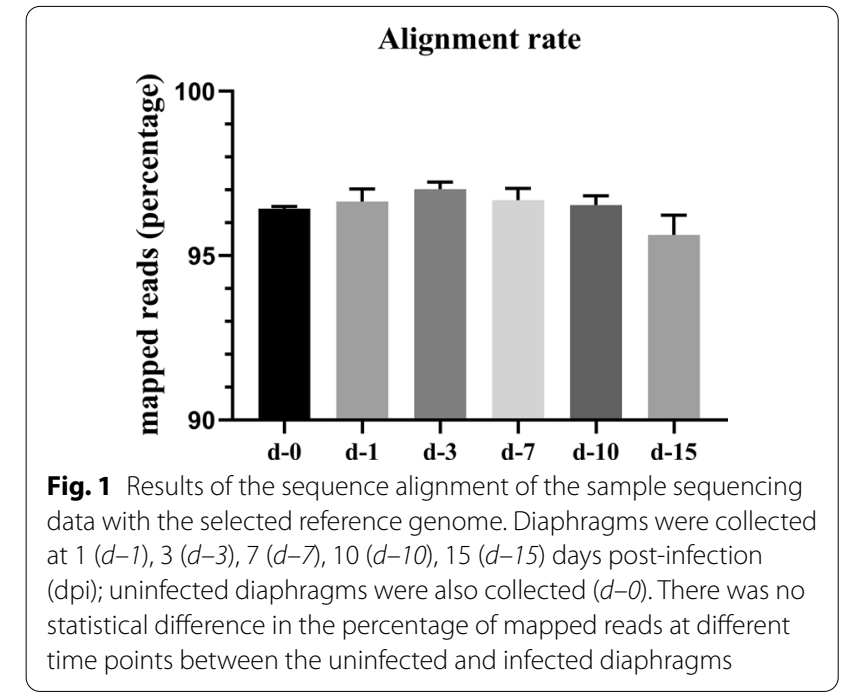

genes were uniformly up- or downregulated at all 5 time points ( 6 genes upregulated at all time points and 5 genes downregulated at all time points; see Additional file 2: Table S2).
Differential expression analysis across time points was conducted to explore statistical differences in gene expression over time. Comparisons between subsequent time points demonstrated substantial numbers of DEGs from 1 to $3 \mathrm{dpi}$ (423 genes) and between 10 and $15 \mathrm{dpi}$ (1098 genes), but notably few significantly differentially expressed genes were observed between 3 and 7 dpi (18 genes) or between 7 and $10 \mathrm{dpi}$ (67 genes) (Fig. 4, bottom panel). This result indicates that the massive initial reaction of the murine muscle cells to Trichinella infection steadied in the later stages of the infection, with few additional changes detected for the period studied, as the infected cells generally maintained their expression pattern. In the late stage of infection, diaphragm DEGs varied dramatically, roughly consistent with the changes of DEGs at each previous time point. These results imply that the response of muscle after NBL entry is not a continuous and steady process with a slow, or drastic, trend, but a process that can show both dramatic changes and then more stable ones, exhibiting a specific rhythm. Except for the rapid changes at the beginning of the infection with the entry of NBL, the response of the host tends to be steady, with sharper changes seen once again at the late stage of infection. 


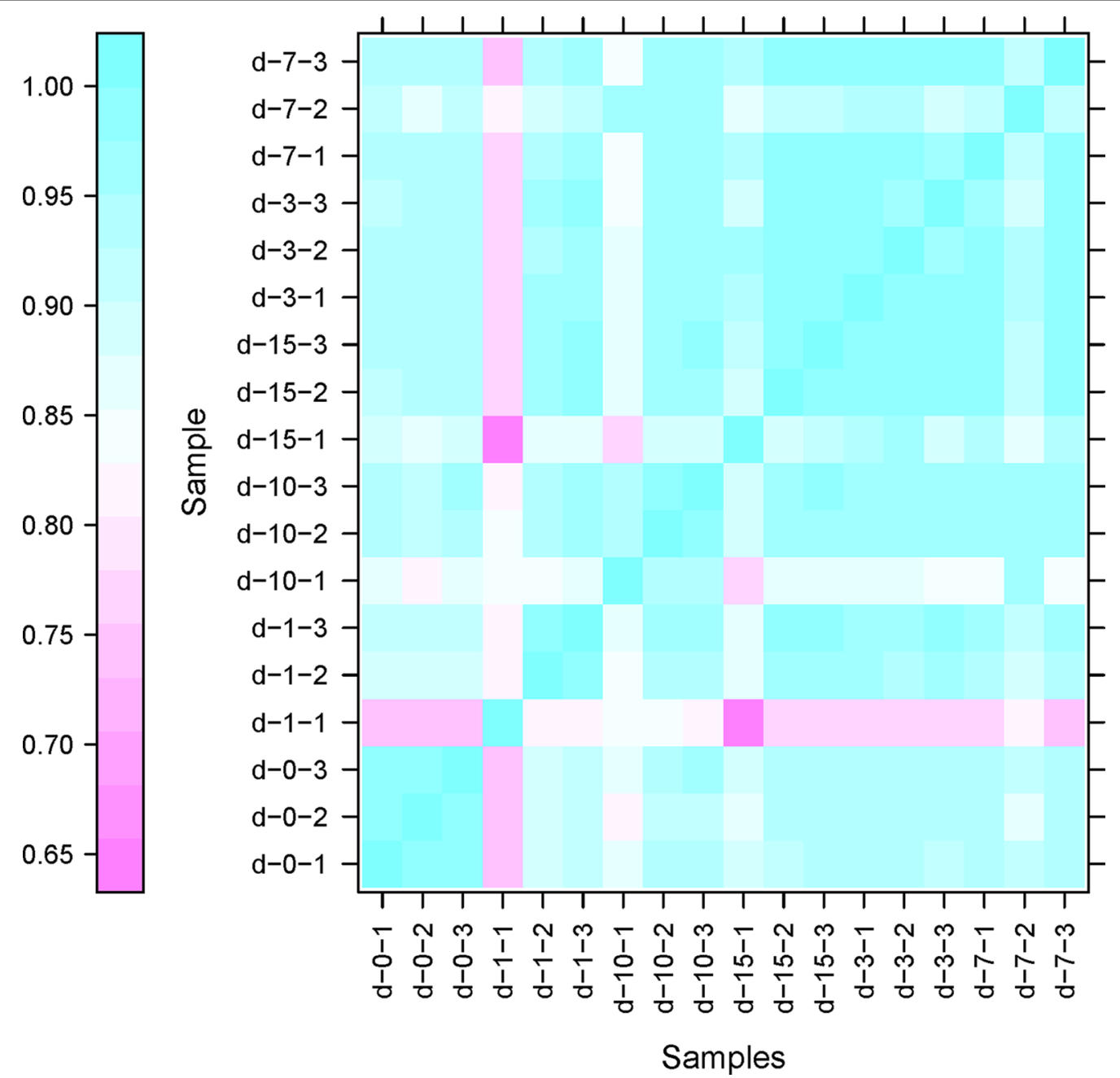

Fig. 2 Global gene expression profiles of murine diaphragm host cells. Pearson's correlation coefficient $\left(R^{2}\right)$ was used as the standard evaluation index of correlation between the biological replicates (replicate number indicated by the final digit of the samples). The closer to 1 the $R^{2}$, the more similar the two replicates. For other abbreviations, see Fig. 1

\section{Eleven genes consistently up- or downregulated at all 5 time points}

The 11 genes that were always either upregulated or downregulated at all 5 time points were: circadianassociated repressor of transcription (Ciart); period circadian clock 2 (Per2); serine (or cysteine) peptidase inhibitor, clade E, member 1 (Serpine1); glycoprotein (transmembrane) $\mathrm{nmb}(\mathrm{Gpnmb})$; ankyrin repeat domain 1 (cardiac muscle) (Ankrd1); ATP synthase 6, mitochondrial (Mtatp6); cartilage intermediate layer protein 2 (Cilp2); tenomodulin (Tnmd); collagen, type II, alpha 1 (Col2a1); Wnt inhibitory factor 1 (Wif1); and Mus_musculus_newGene_8222, which is predicted to be ribosomal protein L10 ( $R p l 10)$. In addition, in spite of the fact that 11 genes were consistently up- or downregulated at all 5 time points, the line graph reflects the disparate variation trend of the expression levels of these genes at each time point (Fig. 5). Among the 6 upregulated genes, expression levels of Ciart, Per2, and Mtatp6 increased steadily then subsequently decreased slightly at $10 \mathrm{dpi}$, while expression levels of the other 3 genes increased sharply on the first day and leveled off at later time points. The other five downregulated genes showed a similar trend, decreasing rapidly at $1 \mathrm{dpi}$ and increasing slowly thereafter without exception.

To verify the genes that were differentially expressed at all 5 time points, the expression levels of 9 genes-Ciart, Per2, Serpine1, Gpnmb, Ankrd1, Cilp2, Tnmd, Col2a1, and Wif1-were analyzed by quantitative real-time PCR. The expression of Ciart, Per2, Cilp2, Tnmd, Col2a1, and Wif1 at each time point post-infection was significantly different from that observed in uninfected diaphragms $(P<0.0001)$. The gene expression trends of the 9 genes 


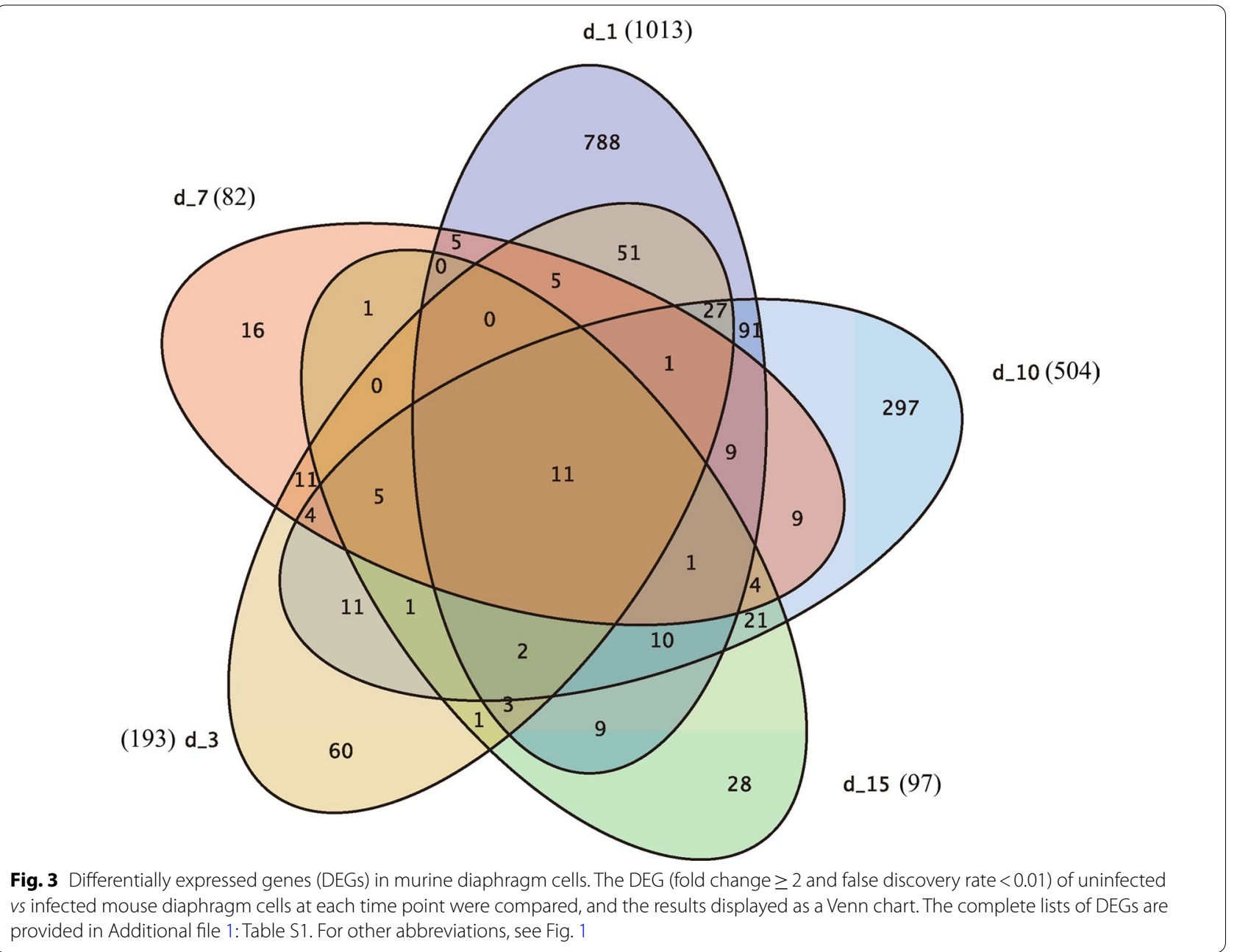

were generally consistent with the sequencing results, confirming the data obtained in the sequencing analysis (Fig. 5).

\section{KEGG annotation and pathway enrichment analysis of DEGs}

The enriched KEGG pathways were employed to analyze the DEG lists to acquire insights into the host response to infection and how it differs between early and later stages of infection. The cellular processes most influenced at each time point were characterized by KEGG pathway enrichment analysis. The KEGG database was employed for functional annotation of DEGs, and the number of genes annotated in the database for each DEG set was 600 (1 dpi), 126 (3 dpi), 53 (7 dpi), 317 (10 dpi) and 73 (15 dpi). Genes that were differentially expressed $>$ twofold were utilized as input with upregulated and downregulated genes considered respectively. The results of this analysis $(q$-value $<0.01)$ are reported in Table 4 . Early on in the infection ( $1 \mathrm{dpi})$, numerous KEGG pathways that were most extremely triggered in infected diaphragms were connected with immune responses, specifically cytokine-cytokine receptor interactions and many signaling pathways, including the tumor necrosis factor, nuclear factor-kappa B, nodulation (NOD)-like receptor, mitogen-activated protein kinase (MAPK), JakSTAT, HIF-1 and adipocytokine ones. KEGG pathways connected with transcriptional misregulation in cancer and microRNAs in cancer are also present in the list of upregulated genes, indicating that the role of Trichinella in cancer warrants further investigation. KEGG pathways downregulated at $1 \mathrm{dpi}$ are mainly related to autoimmune disease, including inflammatory bowel disease (IBD), asthma, rheumatoid arthritis and type I diabetes mellitus. A reduction in the receptors and signaling factors involved in the intestinal immune network for immunoglobulin A production were also observed at $1 \mathrm{dpi}$. Beyond expectation, the results also showed that the invasion of the NBL into muscle cells may be lead to the suppression of intestinal immunity through the 


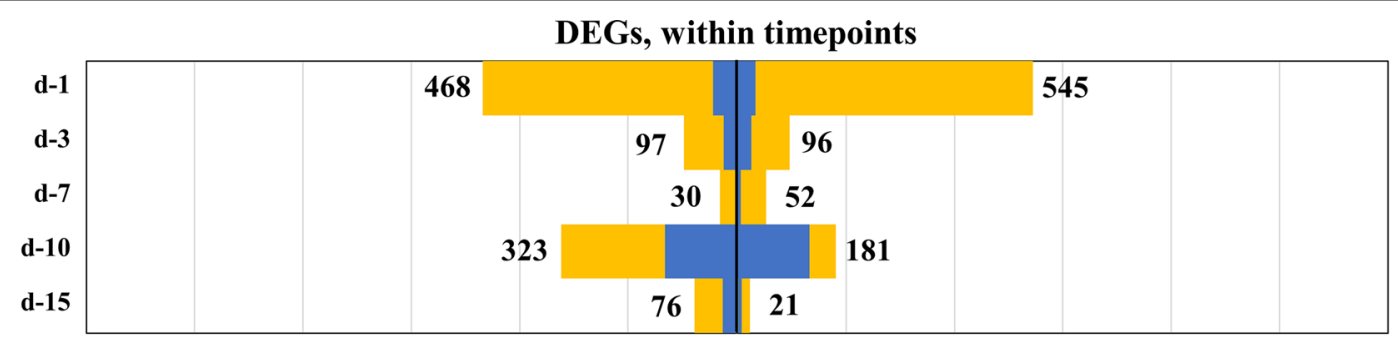

DEGs, between timepoints

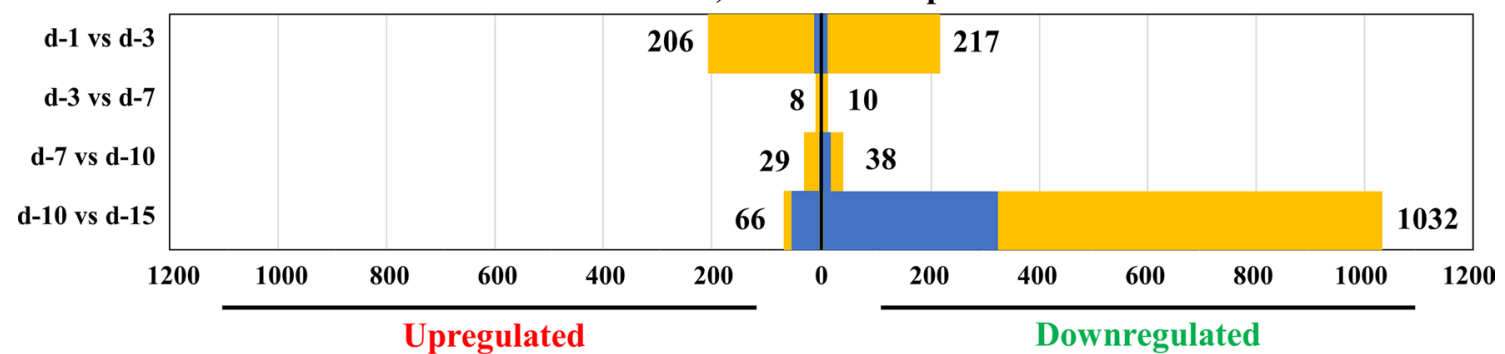

Number of DEGs

Fig. 4 DEGs in murine diaphragm cells. Pairwise comparisons were made to compare DEGs in uninfected vs infected mouse samples at each time point (top) and between time points (bottom). Box length indicates the number of upregulated (left) or downregulated (right) DEGs, with the total number of up- and downregulated genes shown. Genes with > fourfold differential expression (blue) or twofold differential expression (yellow). For abbreviations, see Figs. 1 and 3

whole body mediation network, and in turn promote the survival of adults in the guts. Other pathways that are downregulated include protein digestion and absorption, antigen processing and presentation, cell adhesion molecule and hematopoietic cell lineage pathways.

The enriched KEGG pathways detected at 3, 7, 10 and 15 dpi yielded diverse enrichment patterns compared to those obtained at $1 \mathrm{dpi}$. There were more pathways downregulated than upregulated at every later time point, but no particularly distinct picture was apparent, except for the ECM-receptor interaction, which was nearly always present. Pathways at these four time points were all downregulated, apart from circadian entrainment at $3 \mathrm{dpi}$. Furthermore, the number of enriched KEGG pathways at other time points was significantly lower than that at $1 \mathrm{dpi}$. Similar to the pattern of variation of DEGs, other than the considerable changes at the beginning of the entry of NBL into the host diaphragm, pathway-based enrichment analyses also showed that the muscles were relatively stable before signs of further changes were apparent at the late stage of infection.

A large number of DEGs (1098 genes) were apparent during the transition from 10 to $15 \mathrm{dpi}$; 1032 of these genes were downregulated, and accounted for $94 \%$ of the total DEGs at 10 and $15 \mathrm{dpi}$. We attempted to determine the cause of this massive and extraordinary change. Specifically, we conducted KEGG enrichment analysis of genes downregulated between 10 and 15 dpi (Table 5), and found that almost all the KEGG pathways during the 10-15 dpi transition were related to amino acid metabolism, although no significant increase in these pathways was observed at the previous time points. This findings indicated that a there were fewer DEGs at $15 \mathrm{dpi}$ because T. spiralis had completely developed at this point and stopped absorbing large amounts of energy from the host cells.

\section{GO classification of DEGs}

GO analysis was employed to identify cellular functions and processes that characterize the invasion and survival of T. spiralis in murine myocytes. These functions and processes were compared to the lists of DEGs to characterize how the host adapts to parasite invasion. The GO database was used for the functional annotation of DEGs, and the number of genes annotated in the database for each DEG set was 695 (1 dpi), 141 (3 dpi), 64 (7 dpi), 351 (10 dpi) and 75 (15 dpi). Similar to the number of DEGs and DEGs annotated in the KEGG database at each of these time points, the number of DEGs annotated in the GO database decreased significantly at 3 and $7 \mathrm{dpi}$ compared to $1 \mathrm{dpi}$, suddenly increased sharply at $10 \mathrm{dpi}$ and decreased to a lower level at $15 \mathrm{dpi}$, which was similar to that observed at 3 and 7 dpi. Genes enriched in secondary GO functions against the background of DEGs are shown in Fig. 6, 


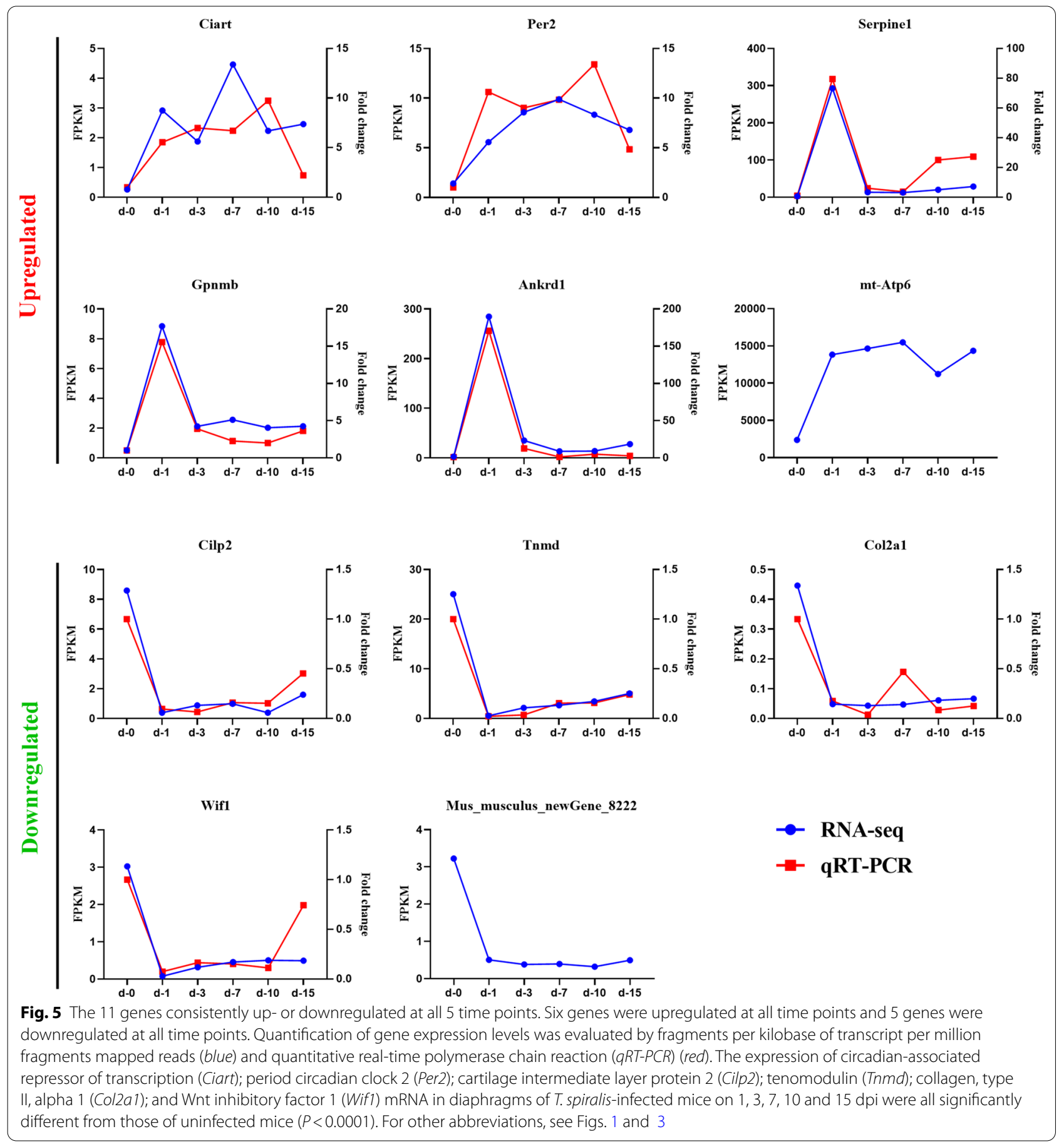

reflecting the status of all secondary functions, and secondary functions with significant proportion differences indicate different enrichment trends of DEGs. In each of the three main categories (biological process, cellular component and molecular function) of the GO classification, cellular process and single-organism process, cell and cell part and binding and catalytic activity were determined to be dominant.

\section{Discussion}

Unlike previous microarray analyses performed on infected tissue or primary myoblast cultures treated with ML-excretory-secretory products, we conducted a more 
Table 4 Kyoto Encyclopedia of Genes and Genomes (KEGG) pathway enrichment analysis of murine diaphragms at 5 time points

\begin{tabular}{|c|c|c|c|c|c|}
\hline $\mathrm{ID}$ & Description & Gene ratio (\%) & Bg ratio $(\%)$ & Enrich. factor ${ }^{a}$ & $q$-value ${ }^{b}$ \\
\hline \multicolumn{6}{|c|}{$d-1$, upregulated } \\
\hline ko04668 & TNF signaling pathway & 11.56 & 1.37 & 8.44 & $2.06 \mathrm{E}-11$ \\
\hline ko04060 & Cytokine-cytokine receptor interaction & 10.98 & 3.19 & 3.45 & 0.000183 \\
\hline ko04064 & NF-kappa B signaling pathway & 7.51 & 1.59 & 4.72 & 0.000183 \\
\hline ko05144 & Malaria & 4.62 & 0.66 & 7.01 & 0.000626 \\
\hline ko05202 & Transcriptional misregulation in cancer & 8.67 & 2.51 & 3.45 & 0.000914 \\
\hline ko04621 & NOD-like receptor signaling pathway & 4.62 & 0.76 & 6.09 & 0.001182 \\
\hline ko05132 & Salmonella infection & 5.20 & 1 & 5.23 & 0.001182 \\
\hline ko05134 & Legionellosis & 4.62 & 0.83 & 5.55 & 0.001781 \\
\hline ko04640 & Hematopoietic cell lineage & 5.78 & 1.37 & 4.22 & 0.002204 \\
\hline ko05206 & MicroRNAs in cancer & 6.36 & 1.79 & 3.55 & 0.004319 \\
\hline ko04933 & AGE-RAGE signaling pathway in diabetic complications & 5.20 & 1.27 & 4.1 & 0.004957 \\
\hline ko04010 & MAPK signaling pathway & 8.67 & 3.27 & 2.65 & 0.007215 \\
\hline ko04380 & Osteoclast differentiation & 5.78 & 1.67 & 3.47 & 0.007614 \\
\hline ko04630 & Jak-STAT signaling pathway & 6.36 & 2.03 & 3.13 & 0.008884 \\
\hline ko04066 & HIF-1 signaling pathway & 5.20 & 1.44 & 3.6 & 0.009357 \\
\hline ko04920 & Adipocytokine signaling pathway & 4.05 & 0.91 & 4.45 & 0.009608 \\
\hline \multicolumn{6}{|c|}{ d-1, downregulated } \\
\hline ko04974 & Protein digestion and absorption & 7.14 & 1.12 & 6.38 & $5.46 \mathrm{E}-06$ \\
\hline ko05150 & Staphylococcus aureus infection & 6.63 & 1.06 & 6.27 & 1.07E-05 \\
\hline ko04612 & Antigen processing and presentation & 6.12 & 1.18 & 5.18 & 0.000166 \\
\hline ko05321 & Inflammatory bowel disease & 5.10 & 0.83 & 6.12 & 0.000166 \\
\hline ko05145 & Toxoplasmosis & 6.63 & 1.47 & 4.52 & 0.000166 \\
\hline ko04512 & ECM-receptor interaction & 5.61 & 1.05 & 5.37 & 0.000166 \\
\hline ko05310 & Asthma & 4.08 & 0.68 & 5.96 & 0.00131 \\
\hline ko04672 & Intestinal immune network for lgA production & 4.59 & 0.90 & 5.12 & 0.001329 \\
\hline ko04514 & Cell adhesion molecules & 7.14 & 2.13 & 3.36 & 0.001439 \\
\hline ko05323 & Rheumatoid arthritis & 5.61 & 1.43 & 3.92 & 0.00198 \\
\hline ko05152 & Tuberculosis & 7.65 & 2.53 & 3.03 & 0.002057 \\
\hline ko05332 & Graft-versus-host disease & 4.08 & 0.78 & 5.21 & 0.002057 \\
\hline ko04640 & Hematopoietic cell lineage & 5.10 & 1.37 & 3.73 & 0.004764 \\
\hline ko04940 & Type I diabetes mellitus & 4.08 & 0.91 & 4.49 & 0.004971 \\
\hline \multicolumn{6}{|c|}{$d-3$, upregulated } \\
\hline ko04713 & Circadian entrainment & 13.89 & 1.19 & 11.62 & 0.004997 \\
\hline \multicolumn{6}{|c|}{ d-3, downregulated } \\
\hline ko04974 & Protein digestion and absorption & 18.42 & 1.12 & 16.45 & 1.37E-05 \\
\hline ko05144 & Malaria & 10.53 & 0.66 & 15.96 & 0.003842 \\
\hline ko05143 & African trypanosomiasis & 10.53 & 0.71 & 14.84 & 0.003842 \\
\hline \multicolumn{6}{|c|}{ d-7, downregulated } \\
\hline ko04512 & ECM-receptor interaction & 23.53 & 1.05 & 22.51 & 0.000396 \\
\hline ko04974 & Protein digestion and absorption & 23.53 & 1.12 & 21.01 & 0.000396 \\
\hline ko04510 & Focal adhesion & 23.53 & 2.60 & 9.05 & 0.00683 \\
\hline ko04710 & Circadian rhythm & 11.76 & 0.35 & 33.76 & 0.009739 \\
\hline \multicolumn{6}{|c|}{ d-10, downregulated } \\
\hline ko04974 & Protein digestion and absorption & 12.90 & 1.12 & 11.52 & $4.08 \mathrm{E}-05$ \\
\hline ko04512 & ECM-receptor interaction & 11.29 & 1.05 & 10.8 & 0.000179 \\
\hline ko04932 & Non-alcoholic fatty liver disease & 12.90 & 1.94 & 6.65 & 0.000855 \\
\hline ko05010 & Alzheimer's disease & 12.90 & 2.24 & 5.76 & 0.001788 \\
\hline ko00190 & Oxidative phosphorylation & 11.29 & 1.77 & 6.39 & 0.001807 \\
\hline
\end{tabular}


Table 4 (continued)

\begin{tabular}{|c|c|c|c|c|c|}
\hline ID & Description & Gene ratio (\%) & Bg ratio (\%) & Enrich. factor ${ }^{\mathrm{a}}$ & $q$-value ${ }^{b}$ \\
\hline ko04151 & PI3K-Akt signaling pathway & 17.74 & 4.62 & 3.84 & 0.001807 \\
\hline ko05012 & Parkinson's disease & 11.29 & 1.84 & 6.13 & 0.001807 \\
\hline ko05016 & Huntington's disease & 12.90 & 2.48 & 5.21 & 0.001807 \\
\hline
\end{tabular}

KEGG pathway analysis identified signaling pathways over-represented in T. spiralis-infected mouse diaphragms $(q$-value $<0.01)$ compared to uninfected controls. Genes that were differentially expressed more than twofold were utilized as input with up- and downregulated genes considered respectively. For each enriched KEGG pathway, the ratio of DEGs in the pathway/all DEGs in all pathways (Gene ratio) and the ratio of genes in the pathway/all genes in all pathways (Bg ratio) are reported

TNF Tumor necrosis factor, ECM extracellular matrix protein, NF nuclear factor, NOD nodulation, $A G E-R A G E$ advanced glycation endproducts-receptor for advanced glycation endproducts, JAK-STAT Janus kinase-signal transducer and activator of transcription pathway, IgA immunoglobulin A, HIF-1 hypoxia-inducible factor 1 , PI3KAkt phosphatidylinositol 3-kinase/protein kinase B

a Enrichment (Enrich.) factors show the proportion of gene ratio to Bg ratio. The bigger the enrichment factor, the more significant the pathway

${ }^{b} q$-value is the $p$-value adjusted by multiple hypothesis testing. The smaller the $q$-value, the more significant the pathway. The DEGs corresponding to the respective enriched KEGG pathway are shown in Additional file 3: Table S3

Table 5 KEGG pathways enriched in murine diaphragms between 10 and 15 dpi

\begin{tabular}{|c|c|c|c|c|c|}
\hline ID & Description & Gene ratio (\%) & Bg ratio (\%) & Enrich. factor & $q$-value \\
\hline ko00040 & Pentose and glucuronate interconversions & 3.75 & 0.51 & 7.35 & 0 \\
\hline ko00260 & Glycine, serine and threonine metabolism & 4.13 & 0.57 & 7.21 & 0 \\
\hline ko00983 & Drug metabolism-other enzymes & 4.69 & 0.73 & 6.38 & 0 \\
\hline ko04610 & Complement and coagulation cascades & 9.19 & 1.14 & 8.03 & $1.36 \mathrm{E}-31$ \\
\hline ko00140 & Steroid hormone biosynthesis & 8.63 & 1.12 & 7.71 & $1.68 \mathrm{E}-28$ \\
\hline ko00053 & Ascorbate and aldarate metabolism & 3.75 & 0.39 & 9.73 & $2.12 \mathrm{E}-14$ \\
\hline ko00380 & Tryptophan metabolism & 3.94 & 0.57 & 6.88 & $1.46 \mathrm{E}-11$ \\
\hline ko00830 & Retinol metabolism & 9.01 & 1.19 & 7.54 & $3.64 \mathrm{E}-11$ \\
\hline ko05204 & Chemical carcinogenesis & 8.44 & 1.27 & 6.65 & 4.65E-11 \\
\hline ko00120 & Primary bile acid biosynthesis & 2.45 & 0.20 & 12.25 & 5.01E-11 \\
\hline ko00982 & Drug metabolism-cytochrome P450 & 6.00 & 0.93 & 6.43 & $9.06 \mathrm{E}-11$ \\
\hline ko00980 & Metabolism of xenobiotics by cytochrome P450 & 5.63 & 0.93 & 6.03 & $9.06 \mathrm{E}-11$ \\
\hline ko03320 & PPAR signaling pathway & 5.44 & 1.13 & 4.804136 & $1.43 \mathrm{E}-10$ \\
\hline ko04146 & Peroxisome & 5.07 & 1.06 & 4.788544 & $6.56 \mathrm{E}-10$ \\
\hline ko00071 & Fatty acid degradation & 3.75 & 0.66 & 5.688697 & $1.26 \mathrm{E}-08$ \\
\hline ko00860 & Porphyrin and chlorophyll metabolism & 3.19 & 0.55 & 5.82445 & $2.38 \mathrm{E}-07$ \\
\hline ko00591 & Linoleic acid metabolism & 3.38 & 0.65 & 5.22 & $6.13 \mathrm{E}-07$ \\
\hline ko00590 & Arachidonic acid metabolism & 4.50 & 1.16 & 3.89 & 1.17E-06 \\
\hline ko00270 & Cysteine and methionine metabolism & 3.19 & 0.63 & 5.03 & $3.31 \mathrm{E}-06$ \\
\hline ko00500 & Starch and sucrose metabolism & 3.38 & 0.76 & 4.45 & 1.07E-05 \\
\hline ko04976 & Bile secretion & 3.56 & 0.88 & 4.03 & $2.58 \mathrm{E}-05$ \\
\hline ko01040 & Biosynthesis of unsaturated fatty acids & 2.25 & 0.36 & 6.24 & $3.02 \mathrm{E}-05$ \\
\hline ko04950 & Maturity onset diabetes of the young & 2.06 & 0.32 & 6.38 & 7.86E-05 \\
\hline ko01230 & Biosynthesis of amino acids & 3.94 & 1.22 & 3.23 & 0.000322 \\
\hline ko00630 & Glyoxylate and dicarboxylate metabolism & 2.06 & 0.37 & 5.53 & 0.000435 \\
\hline ko00340 & Histidine metabolism & 1.88 & 0.32 & 5.80 & 0.000786 \\
\hline ko00220 & Arginine biosynthesis & 1.69 & 0.26 & 6.46 & 0.000854 \\
\hline ko00770 & Pantothenate and CoA biosynthesis & 1.50 & 0.22 & 6.70 & 0.002209 \\
\hline ko00350 & Tyrosine metabolism & 2.25 & 0.52 & 4.31 & 0.002898 \\
\hline ko00410 & beta-Alanine metabolism & 1.88 & 0.40 & 4.71 & 0.006635 \\
\hline
\end{tabular}

KEGG pathway analysis identified pathways over-represented in T. spiralis-infected mouse diaphragms $(q$-value $<0.01)$ between 10 and 15 dpi. Downregulated genes that were differentially expressed more than twofold were utilized as input. The DEGs corresponding to every enriched KEGG pathway are shown in Additional file 4: Table S4

PPAR Peroxisome proliferator-activated receptor, COA coenzyme A; for other abbreviations, see Table 4 


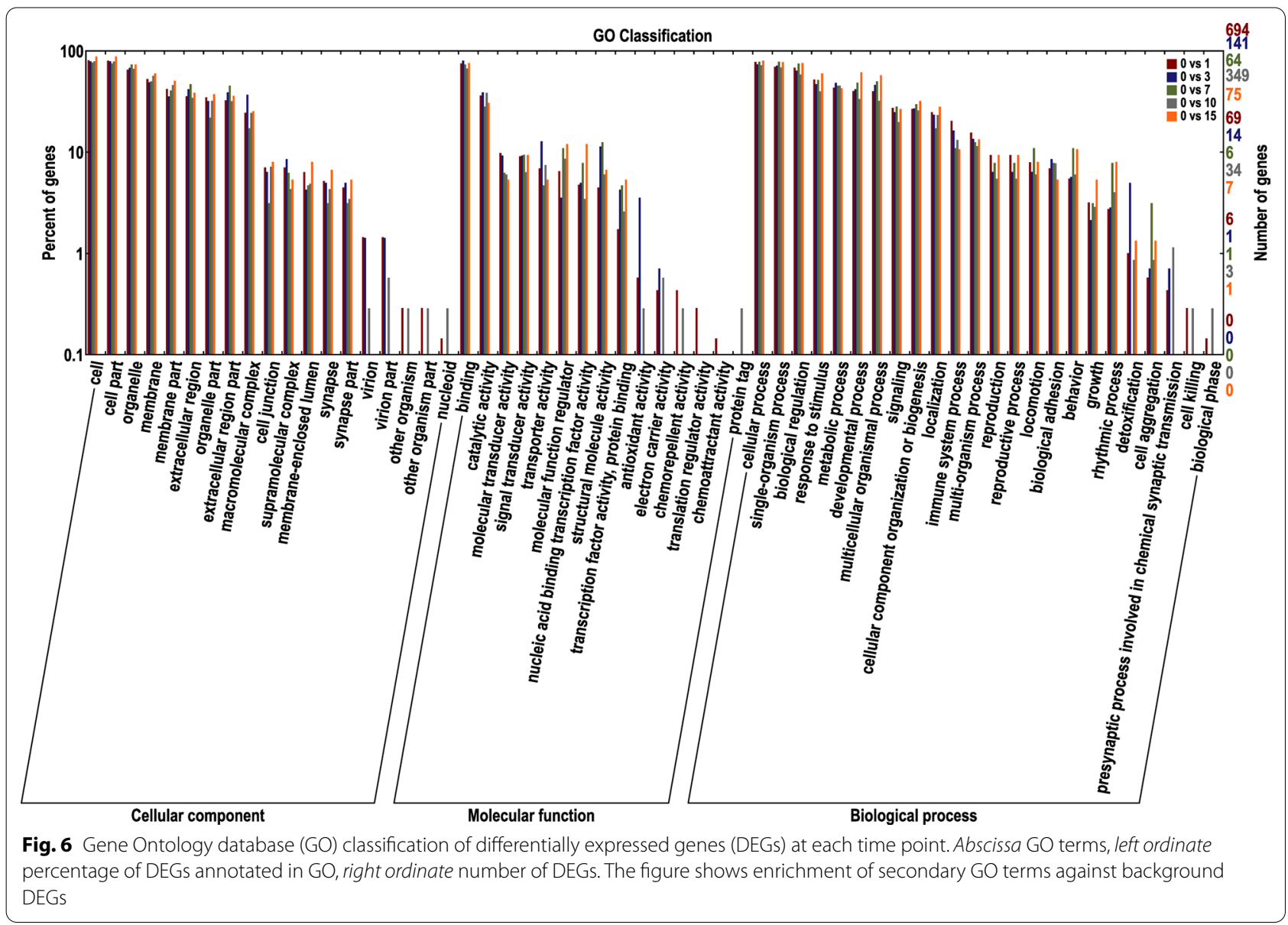

comprehensive and in-depth host transcriptome study to examine the host response to the muscle stage of T. spiralis infection by using advanced RNA-seq technology. In order to maximize the differences in the obtained data, we collected murine diaphragms that had been infected intravenously to enhance differences and to make it easier to discern the host's pivotal functional genes or pathways. By investigating the host transcriptome at different time points, we are able to understand the changing trends of the host response to T. spiralis infection in greater detail. We originally intended to explore host-Trichinella interactions in myocytes through daily dual transcriptome dynamic analysis after NBL invasion. However, this aim could not be achieved due to current technical limitations. The percentage of transcriptome data on Trichinella after in vivo infection was too low to be useful; therefore, only a time-resolved dynamic profile analysis of host transcription levels at the different time points was performed in this study. In addition, there is some, limited agreement between earlier reported DEGs and our dataset, which probably reflects the differences in how these studies were carried out (e.g., the combination of mouse strain and parasite species, tissue sources, and parasite regulation).

Studies have shown that there is biological variability of gene expression in different individuals, i.e. experimental differences due to subgroups of measurements that are not correlated with the fundamental biology of the system being investigated $[15,16]$. To identify DEGs reliably, biological variability should be considered [17]. The most effective and commonly used method for this is to establish biological replicates in the experimental design, i.e. multiple biological samples prepared under the same conditions. Assessment of the relevance of biological replicates is critical to the analysis of transcriptome sequencing data in a project. Prior analyses of high-throughput data, such as those produced using RNA-seq, have demonstrated the need to evaluate and correct biological variability to prevent the misinterpretation of results $[18$, 19].

The results obtained at each time point showed that there was a trend of a rapid rise-decline-rise again in all the transcripts, DEGs and enriched pathways. These findings may change our present understanding of how 
organisms react to Trichinella infection. The way that organisms resist $T$. spiralis, or the effect of Trichinella on organisms, may not be gradual, but erratic. Since NBL were injected into the mice and arrived at the muscles via blood vessels, the physical damage caused by the penetration of the NBL and their secretions would have been extreme stimuli for the muscle cells. Hence, drastic changes in the number of DEGs on the first day after infection are understandable. After this point, Trichinella presumably utilizes its own excretory-secretory products to regulate the host's immune responses, and to make these as stable as possible and suitable for the survival of the parasites. This is thought to explain why there was a subsequent significant decrease in the number of DEGs at 3 and $7 \mathrm{dpi}$. On $10 \mathrm{dpi}$, however, there was a massive increase in the number of DEGs, and we tried to examine the reasons for this from the perspective of the growth and development of the larvae. From the time at which NBL penetrate muscle cells to complete nurse cell formation, the only completely developed organ of $T$. spiralis is the stichosome. As the site where most antigenic components are released, the number of stichocytes in the stichosome is greatest from 6 to $10 \mathrm{dpi}$ [20]. Furthermore, in the early stage of infection in muscle (approximately $10 \mathrm{dpi}$ ), stichocyte-specific antigens begin to be synthesized and are secreted into the surroundings of the nurse cell for the development of nurse-parasite complexes [21]. This phenomenon may account for the sharp change in the number of DEGs at $10 \mathrm{dpi}$. Moreover, by performing KEGG enrichment analysis of downregulated genes between 10 and $15 \mathrm{dpi}$, we could observe that the significantly decreased expression of DEGs from 10 dpi occurred because $T$. spiralis gradually completed its development at this point and stopped absorbing large amounts of energy from the host.

By comparing the differential gene expression libraries obtained from diaphragms at five time points post-NBL invasion, we were able to identify a large number of genes of considerable functional importance with differential expression. Among these genes, only 11 showed consistent tendencies for expression at five time points, suggesting that they may play a pivotal role in host responses to Trichinella infection. Some of these genes also appear to be functionally linked. Notably, host genes related to circadian cycles were first noticed in Trichinella infection. In addition to genes involved in nutrient metabolism, collagen-related gene and Wnt signaling, which were reported to play a part in regulating the formation of nurse cell, are also in this list [22]. The role of serpine1 in combating T. spiralis infection is also worth considering on account of the fact that serine proteases are one of the two prominent excretory-secretory protein families involved in host-parasite interactions in trichinellosis [23].

Per2 and Ciart, first found in mice by Anafi et al. in 2014, actively participate in the regulation of circadian rhythms and are core regulatory elements of the circadian rhythm in mammals [24, 25]. Various physiological systems in the host that could affect parasite life cycles are under circadian control, such as metabolism and immunity $[26,27]$. The effect of immune rhythms on the response to parasitic infections has been elaborated for other parasitic diseases. The infection of mice by Leishmania parasites later in the day induced greater injuries than when infection occurred late in the night, and late-day/early night infection induced a more vigorous increase of innate immune cells [28]. In contrast to the immediate inflammatory response in that Leishmania study, another study showed that the timing of Trichuris infection resulted in a difference in the timing of worm exclusion and antibody induction several weeks later [29]. Furthermore, numerous instances of parasites influencing their hosts' circadian rhythms have been studied. Human African trypanosomiasis (sleeping sickness) is a parasitic disease that shows the ability of parasites to coordinate host rhythms, as infected patients show confusion in their sleep-wake cycles, which becomes more serious as the disease develops [30]. A thorough analysis of Trypanosoma brucei's capacity to undermine host rhythms has been newly reported [31]. The results of that study showed that the normal circadian rhythms of mice became phase advanced after infection, with eccentric movement happening during the rest phase, which was proposed to be linked to mutations in clock genes only [31]. Despite the fact that there are now many reported instances of the significance of circadian or routine rhythms in host-parasite interactions, only a few studies have reported the impact of Trichinella on host circadian clock regulation. The discovery in this study that infection with Trichinella caused changes in the expression of host circadian genes may improve our knowledge of the circadian regulation of host-Trichinella interactions, and may facilitate the formulation of novel preventive and therapeutic policies for trichinellosis.

When T. spiralis NBL penetrate skeletal muscle, they induce the activation and proliferation of satellite cells, which is part of the injury response in adult skeletal muscle. However, these cells do not differentiate into myotubes; instead, they contribute to nurse cell formation [5]. The process of T. spiralis nurse cell formation shares many characteristics with that of mammalian myocyte repair, specifically regeneration, proliferation and collagen production [22]. As an NBL-stage-specific serine protease-like protein, $T s$-NBLsp may function in the larval invasion of skeletal myocytes and nurse cell formation; in 
support of this, promotive proliferation and upregulation of collagen I and VI expression were observed in our TsNBLsp transfected myoblasts (unpublished data). NBL may stimulate the production of serpine1 in the host by secreting serine and as a consequence of physical trauma. Serpine1, upregulated in some cell models during injury repair, is able to orchestrate wound site stroma and tissue remodeling in the wound microenvironment by restraining plasmin-mediated matrix metalloproteinase (MMP) activation [32]. Additionally, the derepression of plasminmediated MMP activation causes degradation of collagen and its decreased deposition, leading to a diminution of fibrotic matrix accumulation in muscles [33]. Recent discoveries emphasize the significance of MMP activities in cell proliferation, migration, and tissue homeostasis [32]. Among the developmental stages of $T$. spiralis, NBL are the most frail but are most exposed to the host immune system. Serpins can help NBL penetrate host defensive barriers more efficiently and avoid immune attack at the same time.

Nurse cells originate from muscle cells during their regeneration. In skeletal muscle, Wnt signaling is involved in myogenesis and muscle regeneration [34]. In a previous study, the Wnt signaling-related expression level was detected by using quantitative real-time PCR with PCR arrays in addition to an examination of microarray data sets characterizing the nurse cell transcriptome $[9,22]$. Similar to the results of this study, the importance of suppression of Wnt signaling in nurse cells at the plasma membrane level was highlighted by higher upregulation of Wif1 expression in nurse cells compared with $\mathrm{C} 2 \mathrm{C} 12$ myoblasts/myotubes. The genes restraining the canonical Wnt signaling cascade and transmitting the signal of Wnt noncanonical signaling cascades were found to be expressed in nurse cell at high levels, reflecting inhibition of Wnt signaling cascade activity and the predominant character of noncanonical Wnt signaling in the long-term conservation of nurse cell biological functions [22]. Our data further corroborate previous findings that misdifferentiation of infected myofibers and nurse cell functional specificity are regulated by Wnt signaling cascades, according to extrapolation.

In addition to the above, the presence of autoimmune disease-related pathways in downregulated KEGG pathways at $1 \mathrm{dpi}$ attracted our attention. Due to the existence of nurse cells, which occupy an immunologically privileged position, T. spiralis and its products can form an immunomodulatory network that may enable the host to cope with miscellaneous hyperimmune-associated diseases [35]. Trichinella infection causes Th2biased immune responses and suppressive/regulatory pathways that diminish excessive inflammation to promote chronic helminth infection in the host and protect host hypersensitivity from autoimmune disorders. Mice infected with $T$. spiralis exhibited fewer arthritis episodes and observably alleviated pathology of collagen-induced arthritis in contrast to uninfected mice [36]. Recent research indicated that $T$. spiralis extracellular vesicles notably attenuated 2,4,6-trinitrobenzene sulfonic acidinduced colitis in mice [37]. Recombinant serine protease from T. spiralis NBL has also been proven to have a feasible protective impact against IBD [38]. Moreover, the preventive and therapeutic impacts of T. spiralis adult extracts on allergic inflammation in an experimental asthma mouse model and inhibition of autoimmune type 1 diabetes by infection with the gastrointestinal helminth T. spiralis have been proven [39]. Notably, unlike the three other diseases that are regulated by Th1 immune responses, asthma, a chronic inflammatory disorder of the respiratory tract, is closely related to abnormal Th2 cell responses [40]. Therefore, the effect of T. spiralis on these four diseases cannot merely be generalized by the Th2 response due to Trichinella infection. To understand the underlying reasons for this phenomenon, we investigated specific downregulated genes in pathways associated with these four autoimmune diseases and found that the expression levels of class II histocompatibility antigen-related genes (H2-Aa, H2-DMb2, H2-DMa, H2-Eb1, $H 2-A b 1$, and $H 2-D M b 1)$ were significantly downregulated. Unlike antigenic peptides from extracellular bacteria and viruses that affect antigen-presenting cells, Trichinella infection may reduce the activation of Th cells by decreasing the expression of major histocompatibility complex molecules in antigen-presenting cells. Although these four diseases are associated with two different Th responses, $T$. spiralis may play a role in disease by inhibiting Th cell activation and limiting interactions between antigen-presenting and $T$ cells, rather than these subsequently differentiating to each Th phenotype. Understanding the mechanisms and identifying the molecules that regulate the complicated host-Trichinella relation could help us to utilize them in the treatment of various autoimmune and allergic diseases. Further studies on DEGs in the KEGG pathway conducted by our research group may help to elucidate the mechanisms governing Trichinella-induced immunomodulation of host autoimmunity.

Contrary to assumptions, pathways associated with nutrient metabolism reported in other parasites, such as glycolysis/gluconeogenesis, have not been observed to be affected after NBL invasion. Thus, following the entry of NBL, the diaphragms did not seem to undergo metabolic changes that cause an enhancement in glycolysis or the production of ATP. These findings indicate that NBL probably develop by deriving nutrients in other ways, such as autophagy. Genes related with specific cell 
biological changes of infected muscle cells at each time point, such as apoptosis, muscle development, myogenesis and regeneration and cell cycle regulation, are also listed in Additional file 5: Table S5. We found that very few well-known genes were differentially expressed, and that only a few DEGs associated with these biological changes were found in the sequencing results of this study. Moreover, numerous disease-specific KEGG pathways were enriched among up- and downregulated genes covered at various time points in this study (i.e., malaria, salmonella infection, legionellosis, staphylococcus aureus infection, toxoplasmosis, tuberculosis and graft-versushost disease; see Table 4); this has also been observed in host transcriptional profiling of other parasitic infections [41]. The link between these diseases and our dataset may have other notable implications. However, although it is important to underline that small overlaps between the comprehensive DE profiles in this paper and the genes involved in these pathways, as well as the deviations in scope between the lists, block the validity of contrasts to disease KEGG pathways as presently defined, these results still imply a degree of consistency in the effects of different pathogens on these pathways after host infection.

Although abundant novel discoveries were made in this study, there may be many genes or pathways of significance that were not unearthed, since our study had its limitations. Hopefully, our data can be exploited by other researchers investigating $T$. spiralis, or even other parasites, and may pave the way for further research investigating parasite biology and host-parasite interactions.

\section{Conclusions}

We employed host genome sequence information and RNA-seq technology to systematically analyze information on the transcriptome of the host at different time points after infection. The most extensive and dynamic gene expression responses in host diaphragms were observed during early infection $(1 \mathrm{dpi})$. Only 11 genes were consistently up- or downregulated at all 5 time points, and KEGG pathways enriched at different time points exhibited different characteristics. The results presented here on the Trichinella-infected host transcriptome may help to elucidate the regulatory mechanism affecting the growth and development of these parasites and the mechanism underlying the host-parasite interaction.

\section{Supplementary Information}

The online version contains supplementary material available at https://doi. org/10.1186/s13071-021-04624-6.

Additional file 1: Table S1. Complete DEG lists for each time point.

Additional file 2: Table S2. Eleven genes consistently up- or downregulated.

Additional file 3: Table S3. KEGG pathways enriched at 5 time points. Additional file 4: Table S4. Enriched KEGG pathways between 10 and 15 dpi.

Additional file 5: Table S5. Genes related to specific cell biological changes of infected muscle cells.

\section{Abbreviations}

DEG: Differentially expressed gene; dpi: Days post-infection; GO: Gene Ontology Database; IBD: Inflammatory bowel disease; KEGG: Kyoto Encyclopedia of Genes and Genomes; ML: Muscle larvae; MMP: Matrix metalloproteinase; NBL: Newborn larvae; PCR: Polymerase chain reaction; RNA-seq: RNA sequencing.

\section{Acknowledgements}

Not applicable.

\section{Authors' contributions}

The study was conceived and designed by XXH and XLL. XXH, CYL, YFL and, $Y X C$ performed the experiments. XXH and XXL analyzed the data. XXH, CL and YLZ wrote the manuscript. HG, MYL and XB improved the manuscript. All the authors read and approved the final manuscript.

\section{Funding}

This study was supported by the National Natural Science Foundation of China (NSFC 31872467, 31520103916); the Jilin Provincial Science and Technology Development Project (20180520042JH); and the Program for JLU Science and Technology Innovative Research Team.

\section{Availability of data and materials}

All relevant data are included in the paper and its supporting information files, except for the data deposited in the Sequence Read Archive database under the BioProject ID PRJNA670288.

\section{Ethics approval and consent to participate}

All experiments in this study were performed according to the regulations of the Administration of Affairs Concerning Experimental Animals in China. The animal experimental procedure was approved by the Institutional Animal Care and Use Committee of Jilin University (permit no. 20170318).

\section{Consent for publication}

Not applicable.

\section{Competing interests}

The authors declare that they have no competing interests.

\section{Author details}

${ }^{1}$ Key Laboratory of Zoonosis Research, Ministry of Education, Institute of Zoonosis, College of Veterinary Medicine, Jilin University, Changchun 130062, China. ${ }^{2}$ Beijing Hi-Tech Institute, Beijing 100085, China. ${ }^{3}$ Jiangsu Co-innovation Center for Prevention and Control of Important Animal Infectious Diseases and Zoonoses, Yangzhou, Jiangsu, China.

Received: 4 November 2020 Accepted: 3 February 2021

Published online: 01 March 2021 


\section{References}

1. Bai X, Hu X, Liu X, Tang B, Liu M. Current research of trichinellosis in China. Front Microbiol. 2017;8:1472.

2. Pozio E. Trichinella spp. imported with live animals and meat. Vet Parasitol. 2015:213(1-2):46-55.

3. Liu MY, Wang XL, Fu BQ, Li CY, Wu XP, Le Rhun D, et al. Identification of stage-specifically expressed genes of Trichinella spiralis by suppression subtractive hybridization. Parasitology. 2007;134(Pt 10):1443-55.

4. Gottstein B, Pozio E, Nöckler K. Epidemiology, diagnosis, treatment, and control of trichinellosis. Clin Microbiol Rev. 2009;22(1):127-45.

5. Wu Z, Nagano I, Takahashi Y. Trichinella: what is going on during nurse cell formation? Vet Parasitol. 2013;194(2-4):155-9.

6. Jasmer DP. Trichinella spiralis infected skeletal muscle cells arrest in G2/M and cease muscle gene expression. J Cell Biol. 1993;121(4):785-93.

7. Park MK, Cho MK, Kang SA, Kim BY, Yu HS. The induction of the collagen capsule synthesis by Trichinella spiralis is closely related to proteaseactivated receptor 2. Vet Parasitol. 2016;230:56-61.

8. Wu Z, Nagano I, Boonmars T, Takahashi Y. A spectrum of functional genes mobilized after Trichinella spiralis infection in skeletal muscle. Parasitology. 2005:130(Pt 5):561-73.

9. Hernández-Ancheyta L, Salinas-Tobón MDR, Cifuentes-Goches JC, Hernández-Sánchez J. Trichinella spiralis muscle larvae excretory-secretory products induce changes in cytoskeletal and myogenic transcription factors in primary myoblast cultures. Int J Parasitol. 2018;48(3-4):275-85.

10. Wu J, Tian L, Yu X, Pattaradilokrat S, Li J, Wang M, et al. Strainspecific innate immune signaling pathways determine malaria parasitemia dynamics and host mortality. Proc Natl Acad Sci USA. 2014;111(4):E511-20.

11. Wang $\mathrm{CH}$, Bell RG. Trichinella spiralis: vascular recirculation and organ retention of newborn larvae in rats. Exp Parasitol. 1986;62(3):430-41.

12. Dvinge $H$, Bertone P. HTqPCR: high-throughput analysis and visualization of quantitative real-time PCR data in R. Bioinformatics. 2009:25(24):3325-6.

13. Pfaffl MW. A new mathematical model for relative quantification in realtime RT-PCR. Nucleic Acids Res. 2001;29(9):e45.

14. Shen S, Park JW, Lu Z, Lin L, Henry MD, Wu YN, et al. rMATS: robust and flexible detection of differential alternative splicing from replicate RNAseq data. Proc Natl Acad Sci USA. 2014;111(51):E5593-601.

15. Elowitz MB, Levine AJ, Siggia ED, Swain PS. Stochastic gene expression in a single cell. Science. 2002;297(5584):1183-6.

16. Anders $S$, Reyes $A$, Huber W. Detecting differential usage of exons from RNA-seq data. Genome Res. 2012;22(10):2008-17.

17. Pertea M, Pertea GM, Antonescu CM, Chang TC, Mendell JT, Salzberg SL. StringTie enables improved reconstruction of a transcriptome from RNAseq reads. Nat Biotechnol. 2015;33(3):290-5.

18. Dillon LA, Okrah K, Hughitt VK, Suresh R, Li Y, Fernandes MC, et al. Transcriptomic profiling of gene expression and RNA processing during Leishmania major differentiation. Nucleic Acids Res. 2015;43(14):6799-813.

19. Leek JT, Scharpf RB, Bravo HC, Simcha D, Langmead B, Johnson WE, et al. Tackling the widespread and critical impact of batch effects in highthroughput data. Nat Rev Genet. 2010;11(10):733-9.

20. Despommier DD, Müller M. The stichosome and its secretion granules in the mature muscle larva of Trichinella spiralis. J Parasitol. 1976;62(5):775-85.

21. Despommier DD, Gold AM, Buck SW, Capo V, Silberstein D. Trichinella spiralis: secreted antigen of the infective L1 larva localizes to the cytoplasm and nucleoplasm of infected host cells. Exp Parasitol. 1990;71(1):27-38.

22. Dabrowska M, Skoneczny M, Zielinski Z, Rode W. Wnt signaling in regulation of biological functions of the nurse cell harboring Trichinella spp. Parasites Vectors. 2016:9(1):483.
23. Liu X, Song Y, Jiang N, Wang J, Tang B, Lu H, et al. Global gene expression analysis of the zoonotic parasite Trichinella spiralis revealed novel genes in host parasite interaction. PLoS Negl Trop Dis. 2012;6(8):e1794

24. Anafi RC, Lee Y, Sato TK, Venkataraman A, Ramanathan C, Kavakli IH, et al. Machine learning helps identify CHRONO as a circadian clock component. PLoS Biol. 2014;12(4):e1001840.

25. Goriki A, Hatanaka F, Myung J, Kim JK, Yoritaka T, Tanoue S, et al. A novel protein, $\mathrm{CHRONO}$, functions as a core component of the mammalian circadian clock. PLoS Biol. 2014;12(4):e1001839.

26. Bass J. Circadian topology of metabolism. Nature. 2012;491(7424):348-56.

27. Tognini P, Murakami M, Sassone-Corsi P. Interplay between microbes and the circadian clock. Cold Spring Harb Perspect Biol. 2018;10(9):a028365.

28. Laranjeira-Silva MF, Zampieri RA, Muxel SM, Floeter-Winter LM, Markus RP. Melatonin attenuates Leishmania (L.) amazonensis infection by modulating arginine metabolism. J Pineal Res. 2015;59(4):478-87.

29. Hopwood TW, Hall S, Begley N, Forman R, Brown S, Vonslow R, et al. The circadian regulator BMAL1 programmes responses to parasitic worm infection via a dendritic cell clock. Sci Rep. 2018;8(1):3782.

30. Carvalho Cabral P, Olivier M, Cermakian N. The complex interplay of parasites, their hosts, and circadian clocks. Front Cell Infect Microbiol. 2019;9:425

31. Rijo-Ferreira F, Carvalho T, Afonso C, Sanches-Vaz M, Costa RM, Figueiredo LM, et al. Sleeping sickness is a circadian disorder. Nat Commun. 2018;9(1):62.

32. Simone TM, Higgins CE, Czekay RP, Law BK, Higgins SP, Archambeault J, et al. SERPINE1: a molecular switch in the proliferation-migration dichotomy in wound-"activated" keratinocytes. Adv Wound Care. 2014;3(3):281-90

33. Flevaris $P$, Vaughan $D$. The role of plasminogen activator inhibitor type-1 in fibrosis. Semin Thromb Hemost. 2017;43(2):169-77.

34. Otto A, Schmidt C, Luke G, Allen S, Valasek P, Muntoni F, et al. Canonical Wnt signalling induces satellite-cell proliferation during adult skeletal muscle regeneration. J Cell Sci. 2008;121(17):2939-50.

35. Sofronic-Milosavljevic L, Ilic N, Pinelli E, Gruden-Movsesijan A. Secretory products of Trichinella spiralis muscle larvae and immunomodulation: implication for autoimmune diseases, allergies, and malignancies. J Immunol Res. 2015;2015:523875.

36. Cheng $Y$, Zhu X, Wang $X$, Zhuang $Q$, Huyan $X$, Sun $X$, et al. Trichinella spiralis infection mitigates collagen-induced arthritis via programmed death 1-mediated immunomodulation. Front Immunol. 2018;9:1566.

37. Yang Y, Liu L, Liu X, Zhang Y, Shi H, Jia W, et al. Extracellular vesicles derived from Trichinella spiralis muscle larvae ameliorate TNBS-induced colitis in mice. Front Immunol. 2020;11:1174.

38. Pang J, Ding J, Zhang L, Zhang Y, Yang Y, Bai $X$, et al. Effect of recombinant serine protease from adult stage of Trichinella spiralis on TNBS-induced experimental colitis in mice. Int Immunopharmacol. 2020;86:106699.

39. Sun S, Li H, Yuan Y, Wang L, He W, Xie H, et al. Preventive and therapeutic effects of Trichinella spiralis adult extracts on allergic inflammation in an experimental asthma mouse model. Parasites Vectors. 2019;12(1):326

40. Trujillo-Vargas CM, Werner-Klein M, Wohlleben G, Polte T, Hansen G, Ehlers $\mathrm{S}$, et al. Helminth-derived products inhibit the development of allergic responses in mice. Am J Respir Crit Care Med. 2007;175(4):336-44.

41. Dillon LA, Suresh R, Okrah K, Corrada Bravo H, Mosser DM, El-Sayed NM. Simultaneous transcriptional profiling of Leishmania major and its murine macrophage host cell reveals insights into host-pathogen interactions. BMC Genomics. 2015;16:1108.

\section{Publisher's Note}

Springer Nature remains neutral with regard to jurisdictional claims in published maps and institutional affiliations. 